\title{
LAS REVISTAS CIENTIFICAS: EL CENTRO Y LA PERIFERIA
}

\section{Evaristo Jiménez Contreras*}

Resumen: Se analiza la composición por paises del fondo de Revistas de SCI para establecer el número de las revistas pertenecientes a Francia, Italia y España y su evolución dentro de este conjunto entre los años 1976-90. Igualmente se estudian los datos relativos a la producción de estos países. Por último se apuntan las posibles razones que explican la evolución opuesta de los valores correspondientes a uno y otro grupo.

Palabras clave: Revistas científicas, centro de avance científico, Francia, Italia, España.

\begin{abstract}
SCI journal holding by country is analysed to establish the number of journals of French, Italian and Spanish origin and their development in the period 1976-90. Similarly, data pertaining to these countries production is studied. Finally, the possible reasons which explain the opposing evolution of the corresponding values to one and the other group are outlined.
\end{abstract}

Keywords: Scientific journals, world scientific centre, France, Italy, Spain.

\section{Introducción}

La Ciencia, tal y como se concibe desde hace ya siglos, es un proceso que sólo culmina cuando se publican los resultados de una investigación. El progreso científico se cimenta en la posibilidad de conocer y contrastar lo que los investigadores producen; y este examen continuado, con la aceptación o rechazo de lo que se da a conocer, constituye la dinámica habitual del quehacer científico. En este contexto, el modo en que circula el conocimiento y las formas de acceso a los "productos científicos» son aspectos que pueden condicionar decisivamente el propio desenvolvimiento de la Ciencia. Como postula Brookes en su famosa ecuación, la información sobre el conocimiento modifica el propio conocimiento (1).

El procedimiento habitual para dar a conocer cualquier investigación es, al menos en el campo de las ciencias experimentales, la revista.

Tan antiguas como la propia Ciencia moderna, las revistas científicas ocuparon un lugar subsidiario en este proceso de la comunicación hasta bien entrado el siglo XIX. Sin embargo, a medida que se incrementaban y asentaban las estructuras sociales y académicas de la actividad científica y a medida que el ritmo de desarrollo y la propia especialización dejaban sentir sus efectos, las revistas comenzaron a jugar el papel protagonista que hoy desarrollan.

Los estudios sobre la Ciencia contemporánea, sus características, dinámica, etcétera, descansan en un $95 \%$ en lo que sale a la luz a través de las revistas o en lo que las bases de datos reflejan de su contenido. Ahora bien, si la transferencia del conocimiento y el comportamiento general de la Ciencia han dado origen a ingentes cantidades de estudios, las revistas como tales no suscitan la misma atención pese a contener elementos que, por sí mismos, pueden decidir el mayor o

* Escuela Universitaria de Biblioteconomia y Documentación. Universidad de Granada. Recibido 22-1-92 
menor grado de éxito (es decir, difusión) de los trabajos que los investigadores difunden a través suyo.

En el presente trabajo nos vamos a ocupar de este vehículo, de algunos factores que parecen incidir en su éxito y las consecuencias que de ello pueden derivarse.

\section{Ambito documental}

El marco elegido para este estudio ha sido la base de datos Science Citation Index (SCI), producida por el Institute for Scientific Information, en versión para CD-ROM, y algunas de sus series complementarias como el Journal Citation Reports y directorios (Who is Publishing in Science y Current Bibliographic Directory).

Digamos para situar la cuestión que, en la actualidad, los estudios de Ciencimetría e Informetría en general se hacen en buena medida a partir de los datos que suministra esta base, que se ha convertido en una referencia internacional en lo que al estudio de la Ciencia experimental internacional concierne; por ello tiende a otorgarse a los datos que suministra un valor absoluto que éstos en realidad no poseen. Su éxito, por así decirlo, reposa en dos hechos: por un lado, en los trabajos de $\mathbf{E}$. Garfield sobre la citación en las revistas científicas $(2,3)$ sobre los que se fundamenta la información que proporciona el SCI; por otro, en que el fondo de revistas que indiza esta base no es sólo, presuntamente, el más representativo de la Ciencia internacional, sino que es, sin duda, el de mayores dimensiones y además multidisciplinar. Su presentación específicamente bibliométrica hace además bastante cómoda y precisa la recopilación de información.

A partir de los datos que suministra esta base se han reunido los correspondientes a la producción anual total por paises desde 1976 a 1990 de los principales productores y la información sobre la procedencia geográfica de las revistas que componen el fondo, con la intención de examinar dentro de este conjunto el caso particular de los países mediterráneos occidentales. Por último, se han recogido los valores del factor de impacto de las revistas francesas, italianas y españolas en dos campos científicos: Farmacología y Bioquímica (Fisiología en el caso de España, por carecer de representación en el campo de la Bioquímica).

\section{Centro y periferia de la Ciencia}

Desde los primeros años cincuenta, los historiadores de la Ciencia vienen desarrollando el concepto de "Centro mundial del avance científico" (CA) (4) que se caracterizaría por generar casi la tercera parte del conocimiento conquistado hasta hoy en la totalidad de los espacios científicos y tecnológicos. Este centro está ubicado desde el final de la Primera Guerra Mundial en los EE.UU., y se habría desplazado en los trescientos años anteriores desde Italia (1540-1610), a Inglaterra (1660-1720), después a Francia (1770-1830) y más tarde a Alemania (1840-1914). Durante cada uno de estos períodos, los restantes sistemas nacionales productores de ciencia habrían mantenido conexiones funcionales más o menos sólidas y permanentes con el CA y, en conjunto, pertenecerian a la "Periferia científica» en la medida en que necesitaran importar del CA «de turno» conceptos, métodos, 
medios, orientaciones, procedimientos o instrumentos para contribuir al avance común y que no fueran capaces de generar por sí mismos.

Pues bien, en lo que a nosotros respecta, el CA actual se hallaría físicamente reflejado por las fuentes documentales que utilizan las bases de datos bibliográficos automatizadas norteamericanas y especialmente, por su carácter multidisciplinar, las de la base SCI. La integración funcional de un país determinado en el CA dependería del volumen de su literatura presente cada año en los fondos documentales de aquellas bases, o alternativamente, del número de sus autores censados. Esta integración, considerada desde la perspectiva documental, tiene lugar a través de dos vías complementarias: Una, representada por las revistas del pais en cuestión incorporadas al fondo documental de las bases; la otra, constituida por las revistas de otras nacionalidades (EE.UU. y resto de países) incluidas también en los fondos, $y$ en las que han publicado autores de la comunidad científica nacional en cuestión.

Ni que decir tiene que desde el punto de vista de la producción por países, este centro científico está constituido en su mayor parte por un pequeño grupo de naciones que aporta la mayor parte de la producción mundial. Estos países pertenecen todos a la órbita occidental (con la excepción de la antigua Unión Soviética) y están encabezados por los EE.UU., que suponen por sí solos alrededor de un tercio del total recogido por la base; seguidos del Reino Unido, Alemania, Japón, la antigua URSS, Francia, Canadá, etc. En realidad, aunque dichas cifras no son absolutas, reflejan de un modo aproximado el orden económico mundial, que se corresponde más o menos con el científico, pero sobre todo reflejan la creciente integración en un conjunto homogéneo de las comunidades científicas de los países mencionados, que emplean los mismos canales de difusión de sus trabajos y simultáneamente se nutren de las mismas fuentes.

Donde las cosas distan mucho de funcionar igual es en el caso de las revistas. Como hemos dicho, la aportación de los científicos de cada país se hace a través de un conjunto de revistas de estos mismos países o eventualmente de las revistas de otros países integradas en el fondo. Dicho en otras palabras, los artículos de la comunidad francesa serían difundidos en principio a través de las revistas francesas o a través de las de otros países integradas en la base. Es este el aspecto en el que vamos a incidir porque lo cierto es que, como veremos, en el caso de las revistas no sólo no se está produciendo un movimiento de integración similar al descrito para los científicos sino que por el contrario se está creando una periferia incluso entre las revistas que pertenecen a países integrados en el centro científico internacional.

\section{Análisis de los datos}

A partir de la situación descrita sobre la dinámica de la producción científica y el papel fundamental que la base de datos SCI juega en lo que al estudio de la Ciencia se refiere podemos pasar a valorar los datos reunidos.

Es sabido, y ha sido abundantemente contrastado, que los países de la órbita occidental son los mayores contribuidores netos al total científico mundial y además se comportan de una manera bastante coherente entre ellos. En este conjunto, en la tabla I, observamos como los mediterráneos estudiados, Francia, 
Tabla I

Producción de los países mediterráneos en relación a los EE.UU. 1976/90

\begin{tabular}{lccccccccc}
\hline & 1976 & 1978 & 1980 & 1982 & 1984 & 1986 & 1988 & 1990 \\
\hline EE.UU. & 100 & 100 & 100 & 100 & 100 & 100 & 100 & 100 \\
Francia & 12,8 & 12,5 & 13,9 & 12,4 & 11,9 & 13,2 & 13,6 & 12,9 \\
Italia & 4,9 & 5,2 & 5,4 & 5,9 & 6,2 & 6,3 & 6,9 & 7,4 \\
España & 1,4 & 1,5 & 1,4 & 1,8 & 2,0 & 2,6 & 3,0 & 3,8 \\
\hline
\end{tabular}

Fuente: Who is publishing in Science, Current bibliographic Directory hasta 1980 y SCI, versión CD-ROM, hasta 1990. Philadelphia, ISI.

Italia y España, han desarrollado un proceso de integración creciente en el conjunto internacional desde la fecha de inicio de este examen, 1975, con una tasa de crecimiento positiva prácticamente constante respecto a los totales anuales. En otras palabras, la Ciencia de estos países, en términos de producción dentro del ámbito del SCI, mantiene un grado de presencia creciente - que coincide grosso modo con otros parámetros de análisis como pueden ser su PIB-o, como en el caso de España, ha alcanzado en estos años esta posición. Otra cosa es el tamaño de la misma, muy modesto, en comparación con el gigante americano. Para representar de un modo más gráfico ambas ideas los incrementos se han convertido en porcentajes respecto a un total (100) que representa la producción estadounidense de cada año; mientras que en la figura 1 se refleja el incremento en términos absolutos de la producción.

Figura 1

Produccion internacional $\mathbf{1 9 7 6 - 9 0}$
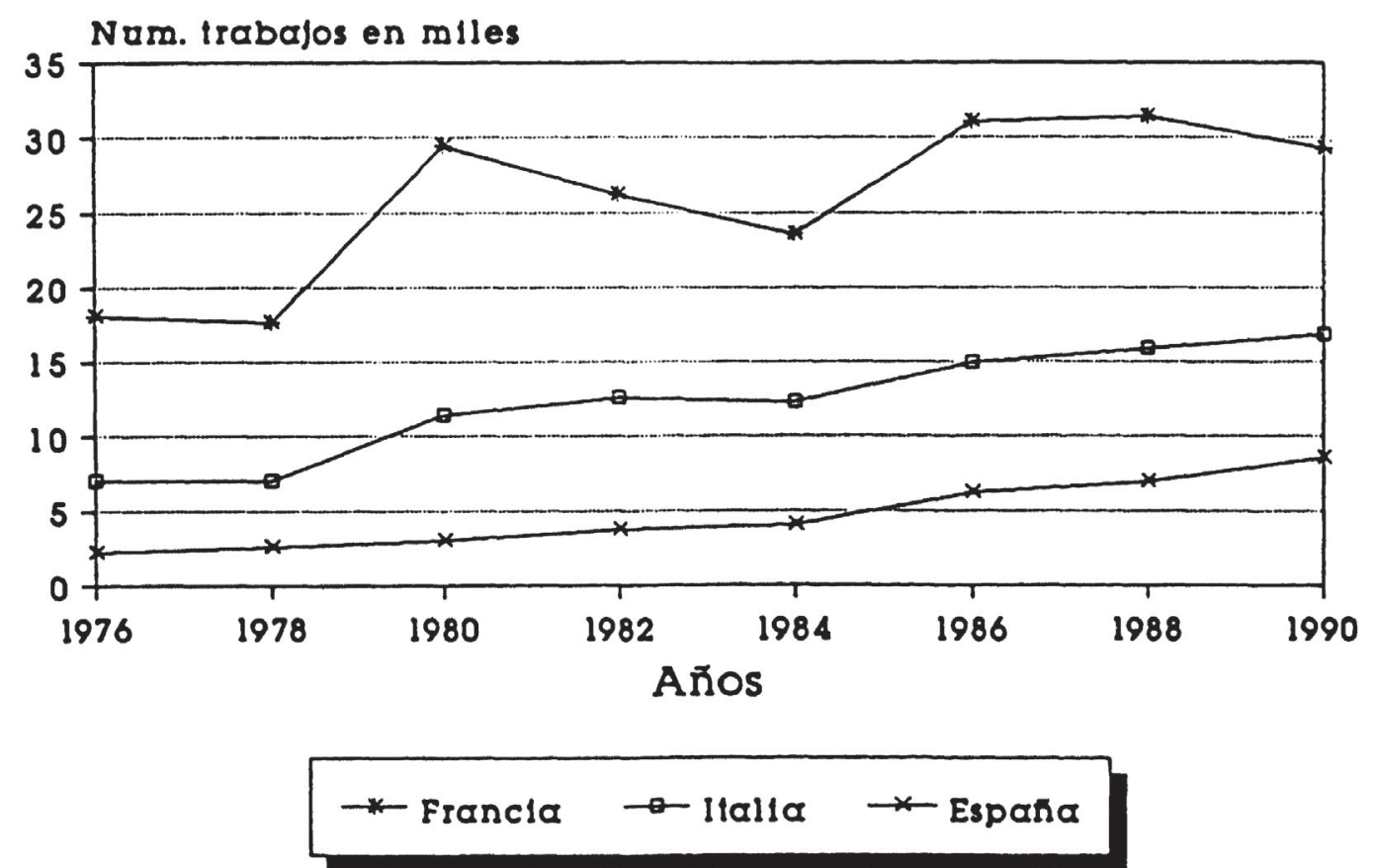

Fuonie: SCl (version CD-ROM) Y WIPIS para los datos anteriores a 1980 
Como dijimos, esta producción, al menos a priori, procedería fundamentalmente de las revistas del mismo país recogidas en la base y secundariamente de las revistas de los restantes países. Eso sería así si el incremento de la producción de un país arrastrase o fuera consecuencia del incremento en el número de revistas de ese mismo país en el fondo. En teoría, en el conjunto de las revistas, se produciría un agrupamiento de las mejores de cada país cuyo número dependería del volumen y recursos de la comunidad científica nacional, y debería existir una cierta correlación entre la comunidad científica integrada en el CA y el número de revistas recogidas por la base. Como muestra la figura 2, según los hechos observados en el fondo documental del ISI, dicho movimiento no sólo no se produce sino que está sucediendo, en el caso de los países mediterráneos mencionados, exactamente lo contrario. La representación de revistas francesas e italianas, cauce natural de difusión de los trabajos de las respectivas comunidades, no ha dejado de menguar en este mismo período. Francia ha pasado de 140 revistas en 1976 a 92 en 1990, con una pérdida promedio de 3,2 revistas anuales, lo que supone que su fondo ha pasado de representar más del $5 \%$ del total en el SCI a menos del $3 \%$, y en conjunto ha perdido prácticamente un tercio de su representación. Italia ha sufrido un proceso similar, como se puede observar en el menciona-

Figura 2

Fondo de revistas SCI 1976-90

Num. revistas

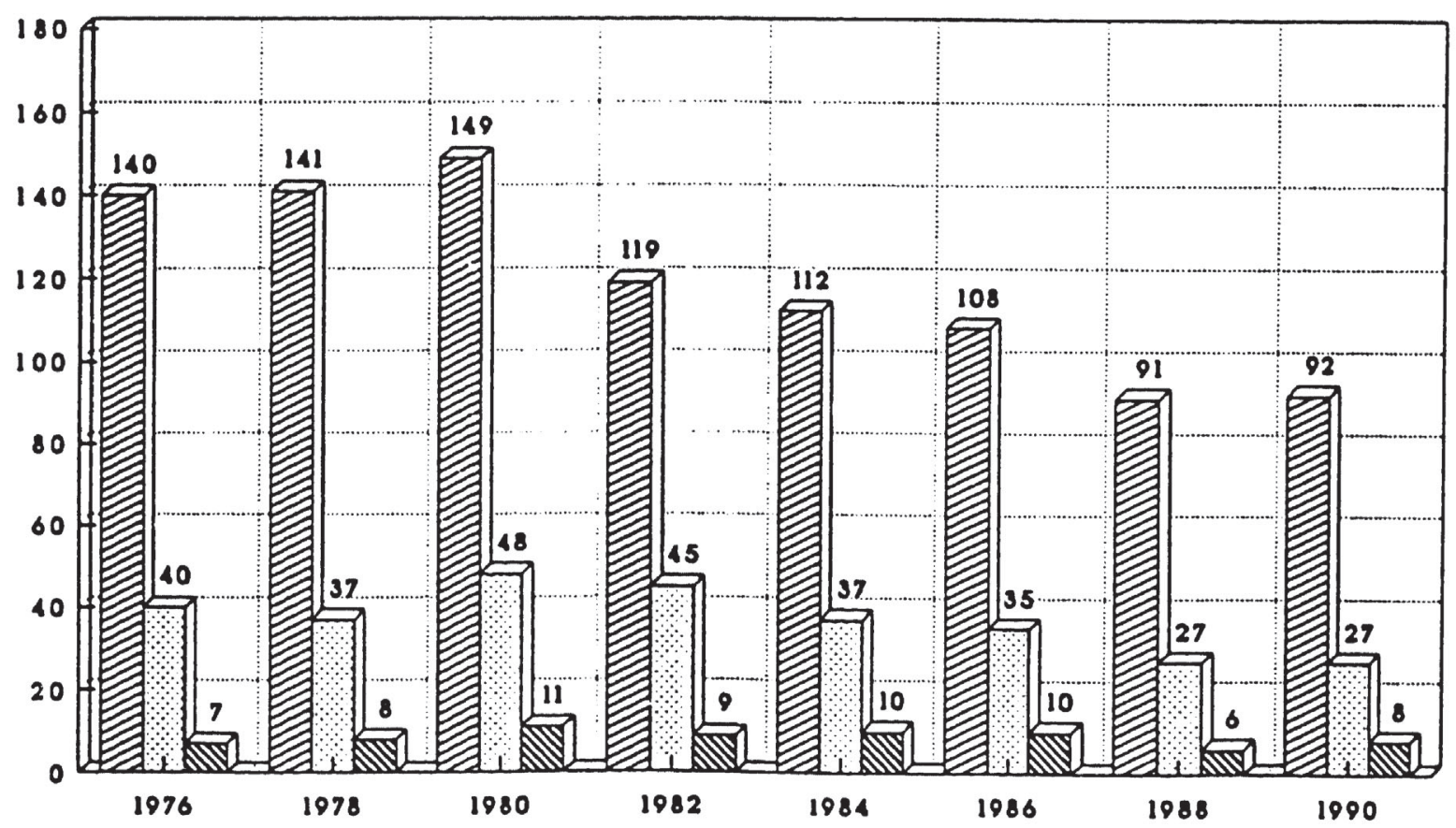

Drancla Ellalla Espata

Fuonto SCI GUIDE $1976-1990$ 
do cuadro, con una pérdida total equivalente de otro tercio. En realidad la situación es aún más dramática si se toman solamente las cifras del último decenio, ya que en los primeros años de nuestro examen el crecimiento del fondo de revistas se correspondió con un incremento de la representación mediterránea. En cuanto a España, se ha mantenido en las cifras iniciales prácticamente simbólicas, pese al poderoso incremento de su producción en términos relativos y absolutos.

Por último, en la tabla II podemos observar los valores del factor de impacto de algunas revistas (hemos elegido para ello los campos con mayor número de revistas en la base SCI: Bioquimica y Farmacología. Por carecer de representación en la primera de estas áreas se ha incluido la Fisiología en el caso español). Las revistas de estos países se mantienen permanentemente en unos niveles muy bajos y con tendencia a retroceder, aunque en este caso los datos son muy escasos y sería necesario proceder a un examen más exhaustivo. Una excepción notable a este

Tabla II

Campo científico bioquímica. Población de revistas 128-141 (Fisiología 44-51)

\begin{tabular}{lcccc}
\hline & \multicolumn{2}{c}{ Año 1984} & \multicolumn{2}{c}{ Año 1988} \\
& F. Impacto & Posición & F. Impacto & Posición \\
\hline $\begin{array}{l}\text { Francia } \\
\text { Biochimie }\end{array}$ & 1,665 & & & \\
B. Soc. Ch. Fr. & 0,802 & 106 & 1,504 & 73 \\
J. Chim. PCB & 1,070 & 85 & 0,610 & 123 \\
Italia & & 0,540 & 128 \\
It. J. Bioch & 0,563 & 112 & & 139 \\
B. Mol. Biol. M & 0,433 & 117 & 0,406 & 141 \\
España & & & 0,350 & \\
Rev. Esp. Fis. & 0,328 & 40 & 0,122 & 48 \\
\hline
\end{tabular}

Campo cientifico farmacología. Población de revistas 125-139

\begin{tabular}{lcccc}
\hline & Año 1984 & \multicolumn{2}{c}{ Año 1988} \\
& F. Impacto & Posición & F. Impacto & Posición \\
Francia & & & & \\
J. Pharmacol & 0,720 & 78 & 0,762 & 76 \\
Eur. J. Med-Ch. & 0,723 & 76 & 0,665 & 84 \\
Ann Pharma & 0,305 & 108 & & 122 \\
Biomed Ph & 0,273 & 113 & 0,250 & 115 \\
J. Pharm. B & 0,198 & 118 & 0,338 & 107 \\
Italia & & & & 130 \\
Farmaco & 0,492 & 92 & 0,399 & \\
Eos & 0,105 & 121 & 0,157 & 85 \\
España & & & & \\
Method Find & 0,420 & 100 & 0,656 & \\
\hline
\end{tabular}

Fuente: Journal Citation Reports 1984 y 1988. Philadelphia, ISI 
respecto la representa la única revista española de Farmacología recogida por el SCI, que ha experimentado en los años examinados un importante progreso que se aparta por completo de la dinámica general que muestra el resto.

Se ha demostrado ya (5) que los valores de los factores de impacto están sometidos a una mecánica que no tiene que ver sólo con la cualidad de los contenidos de las revistas, sino más bien con otros elementos ajenos al contenido científico de los trabajos, y concuerda perfectamente con lo expuesto hasta ahora sobre el comportamiento del fondo de publicaciones periódicas del SCI que las revistas de países como Francia, Italia y España estén habitualmente en el fondo de los rankings de impacto. Por la misma razón que las de EE.UU., Reino Unido, Holanda, etc., están habitualmente en cabeza. La progresión en los rankings de una revista ajena a este núcleo de países resulta doblemente dificil, ya que implica avanzar contra una corriente natural que tiende a hacerla retroceder.

\section{Conclusiones}

¿Qué origen y consecuencias tiene esta evidente disparidad entre la evolución de los datos de producción y los del fondo de revistas?

Para empezar debemos recordar que las fuentes utilizadas por la base SCI son en un porcentaje abrumador (superior al $85 \%$ ) revistas que pertenecen a los países de la órbita científica anglosajona: EE.UU., Reino Unido, Holanda y Suiza; el resto, con las excepciones de Alemania y la antigua URSS, tiene una escasa o casi nula representación documental en la base y este desequilibrio se ha acentuado en los últimos años. Cualquier estudio sobre la producción científica internacional debería tener en cuenta ese sesgo inicial a la hora de evaluar los resultados de los países menos representados.

Como consecuencia de todo lo dicho se dibujan dos dinámicas diferentes, más aún, opuestas, una para la producción científica de los países, en la que los occidentales y más concretamente los mediterráneos considerados muestran una creciente integración, y otra radicalmente contraria en el seno de las revistas en la que las de estos últimos países están quedando en la periferia. Entiéndase en este caso la noción de periferia en relación con el conjunto de los principales productores en el seno del SCI. Existiría un tercer grupo de países que nunca han alcanzado una mínima representación ni parece que estén en camino de conseguirlo o cuyo retroceso coincide con un retroceso generalizado de todos sus parámetros (caso de los países del este europeo).

Cuáles son las razones que subyacen en este diferente comportamiento de dos elementos, producción por paises y población de revistas que, aparentemente, deberían estar relacionados.

Es dificil, si no imposible, identificar todas las causas que contribuyen a esta situación, pero podemos intentar arrojar algo de luz en el asunto. Por supuesto no se trata de que haya sencillamente menos revistas de estos paises en el mercado; en los últimos años su población se ha incrementado como puede comprobarse fácilmente. Sin embargo, han sido incapaces de incorporarse o sencillamente mantenerse en el conjunto de las más utilizadas, como apuntan el número total de las mismas recogido en la base y sus factores de impacto. 
Podría tratarse de un problema de falta de calidad generalizada en la investigación que desarrollan las respectivas comunidades científicas de estos países, sin embargo la creciente presencia de trabajos de investigadores franceses, italianos y españoles en la base de datos, a través de revistas que no son las propias, pues éstas están en pleno retroceso, inclina a pensar que tampoco es un problema de calidades. Un conjunto de científicos no va a destinar sistemáticamente sus mejores trabajos a revistas extranjeras y los mediocres a las de su país (sobre todo teniendo en cuenta que éstas siguen siendo internacionales y cuentan con sus correspondientes sistemas de filtro de los trabajos que se les remiten). Aunque sí puede pensarse que, como consecuencia de este estado de cosas, tal fenómeno está empezando a cobrar forma.

Respecto a las barreras lingüísticas, tema que se ha sacado frecuentemente a colación en relación con la falta de difusión de la investigación de los científicos de lengua no anglosajona, hay que decir que en los últimos años las revistas de Francia, Italia y España han empezado a publicar masivamente en inglés hasta el punto de que en muchos casos incluso sus cabeceras han sido traducidas a esta lengua, sin que hasta ahora esta medida haya frenado el retroceso numérico descrito, más bien coincide el retroceso de las revistas con su conversión a la lengua inglesa.

Se suele aducir también, al menos en el caso de España, que las dificultades de distribución, retrasos en la aparición y otros inconvenientes de parecida índole pesarían de modo negativo en los factores de impacto. Probablemente haya mucho de verdad en esta afirmación, pero dudamos que ésta sea la razón que explique el retroceso de las revistas francesas e italianas, más serias en este sentido que las nacionales, y por otra parte, es precisamente España la única que, pese a los inconvenientes mencionados, ha mantenido sus posiciones en lo que al número de revistas presentes en la base se refiere, incluido algún avance como el señalado en Farmacología.

En última instancia sólo el origen nacional de las revistas parece una causa clara de esta situación. El abrumador desequilibrio en favor de las revistas anglosajonas (con las inclusiones ya mencionadas de Holanda, Suiza y algún otro país) fuerza un desequilibrio de la citación a su favor. Las posibilidades del resto de las revistas de otros países están ya muy mermadas en origen por el solo he ho de no pertenecer a este núcleo, por jugar en campo contrario, si utilizamos un s.mil deportivo. Los científicos anglosajones están más dispuestos a leer sus propias revistas que las francesas, italianas, etc., que además tienen factores de impacto bajos. Es un proceso que se alimenta a sí mismo y cuyas consecuencias se manifiestan en el retroceso generalizado de las revistas que no pertenecen a los países del centro.

La internacionalización de la Ciencia se convierte así para Francia, Italia, España y otros países en un proceso de exportación masiva de su producción a revistas extranjeras, hecho ya descrito hace tiempo para el caso español (6) pero que está afectando a las posibilidades mismas de supervivencia de los canales de difusión de estos países, porque es evidente, digámoslo de una vez, que los científicos franceses, italianos y españoles parecen haber decidido hace tiempo enviar sus trabajos a revistas de otros países porque tienen la justa impresión, dada la dinámica descrita, de que las propias no son el canal adecuado para 
difundir su investigación a nivel internacional, incluso que el reconocimiento académico parece estar ligado a la posibilidad de publicar fuera más que a lo que contengan los trabajos publicados.

A estas alturas es casi redundante decir que las ciencias puras y experimentales han desarrollado un vehículo de transferencia de la información altamente normalizado entre cuyas características está la lengua inglesa. Esta situación favorece el flujo informativo de la ciencia internacional y, teóricamente, que los científicos de todos los países, cualquiera que sea su lengua particular, intercambien información. Lo que se está imponiendo igualmente, y esto no parece necesariamente positivo para todos los países, es que el domicilio editorial de las revistas pase necesariamente por el mundo anglosajón. Las comunidades de profesionales de cada país, la estructuración de las políticas científicas, los respectivos panoramas editoriales, etc., pueden verse afectados a muy corto plazo.

Por otra parte las autoridades científicas pueden llegar a partir de estos mismos datos a la conclusión de que no es rentable mantener unas revistas cuyos indices de visibilidad son tan bajos (en las bases americanas) y, tomando la parte por el todo, llegar a la conclusión de que es mejor y más barato abandonar a su suerte a las revistas científicas de su propio pais en vista de los pobres resultados que obtienen y favorecer la exportación de los trabajos de nuestros investigadores. En España esta política ya tiene carta de naturaleza.

\section{Bibliografia}

1. BROOKES, B. C. The foundations of Information Science. Journal of Information Science, 2, 125-33, 209-21, 269-75, 1980; 3, 3-12, 1982.

2. GARFIELD, E. Citation indexes for Science. Science, 122, 108-11, 1955.

3. GARFIELD, E. Citation Analysis as a tool in journal evaluation. Science, 178, 471-78, 1972.

4. HONGZHOU, G. J. Shifting of World's Scientific Center and scientist's social ages. Scientometrics (Amsterdam), 8, 59-80, 1985.

5. FERREIRO, L.; UGENA, S. Citation mechanics. Scientometrics, 24 (1), 151-164, 1992.

6. FERREIRO, L. Cincuenta años de investigación en Física y Química en el edificio Rockefeller de Madrid (1932-82). Madrid, 1982. 